Ter ahert $z$ absor pt $i$ on spectra of L- , D, and $D L-a l$ ani ne and thei $r$ appl i cat i on to det er mi nat $i$ on of enant $i$ onet $r$ i c composi t $i$ on

\begin{tabular}{|l|l|}
\hline 著者 & $\begin{array}{l}\text { YANAGUCH Nar i ko, M YAMARU Fum aki, YAMAMOTO } \\
\text { Kohj i , TAN Nasahi ko, HANGYO Masanor i }\end{array}$ \\
\hline $\begin{array}{l}\text { j our nal or } \\
\text { publ i cat i on t i t l e }\end{array}$ & Appl i ed physi cs I et t er s \\
\hline vol une & 86 \\
\hline number & 5 \\
\hline page r ange & 53903 \\
\hline year & $2005-01$ \\
\hline URL & ht t p: //hdl . handl e. net /10098/3032 \\
\hline
\end{tabular}




\title{
Terahertz absorption spectra of L-, D-, and DL-alanine and their application to determination of enantiometric composition
}

\author{
Mariko Yamaguchi, ${ }^{a}$ Fumiaki Miyamaru, Kohji Yamamoto, \\ Masahiko Tani, and Masanori Hangyo \\ Institute of Laser Engineering, Osaka University, 2-6 Yamadaoka, Suita, Osaka 565-0871, Japan
}

(Received 19 July 2004; accepted 3 December 2004; published online 27 January 2005)

\begin{abstract}
Absorption spectra of polycrystalline L-, D-, and DL-alanine have been measured by the terahertz time domain spectroscopy (THz-TDS) in the frequency range from 10 to $90 \mathrm{~cm}^{-1}$ at room temperature. We observed several absorption bands, which have the large difference between enantiomers (L- and D-alanine) and the racemic compound (DL-alanine) in their peak frequencies. This obvious difference shows that the $\mathrm{THz}$ absorption bands are strikingly sensitive to the crystal structures. This result indicates that the THz-TDS can be used for distinguishing between the enantiomers and the racemic compound. We propose and demonstrate a method to determine the enantiometric composition of amino acids from the $\mathrm{THz}$ absorption spectra. () 2005 American Institute of Physics. [DOI: 10.1063/1.1857080]
\end{abstract}

Various applications of terahertz $(\mathrm{THz})$ electromagnetic waves have been suggested by many researchers, including medical, pharmaceutical, and security applications. ${ }^{1-8}$ Some achievements such as the marker-free DNA sensing, ${ }^{1}$ the diagnosis of skin cancers, ${ }^{2}$ the inspection of explosives ${ }^{3}$ and illegal drugs, ${ }^{4}$ and the degradation diagnosis of polymers ${ }^{5}$ have already been reported. Absorption bands characteristic to specific crystal structures allow the use of the $\mathrm{THz}$ waves for studying polymorphs of medicines. ${ }^{6}$ Absorption spectra in the THz range provide rich information about weak interactions such as inter-ring interactions of the di-saccharide ${ }^{7}$ and intermolecular interactions of the benzoic acid crystal. ${ }^{8}$ It is expected that the $\mathrm{THz}$ waves can be applied to probing the interactions between biomolecules with which their functions work. Not only probing and sensing for biomolecules, the THz waves may be used in medical treatments or therapeutic ways if sufficiently strong $\mathrm{THz}$ sources are available. Recently, mid-infrared light $(\sim 5.75 \mu \mathrm{m})$ from a freeelectron laser was used to dissociate cholesterol esters, ${ }^{9}$ demonstrating that selective excitations of vibrational modes in biological molecule can be used as a tool for medical treatments. In the same analogy, a concept of selective excitation to damage cancer cells by strong $\mathrm{THz}$ beams for remedy has also been proposed. ${ }^{10}$

Amino acids are biologically important materials because they are backbones of proteins and one of the amino acids (L-glutamic acid) has been measured by the $\mathrm{THz}$ spectroscopy. ${ }^{11}$ Amino acids except glycine have two enantiomers (L- and D-amino acids) and living organisms consist of only L-amino acids. Racemic bodies, which contain equal amounts of the two enantiomers, are classified into two groups according to their physical properties. One is the racemic mixture, which consists of polycrystalline enantiomers and has the same physical properties as the enantiomers. The other is the racemic compound, which is crystallized with the pairs of L- and D-amino acid molecules and shows physical properties different from those of individual enantiomers. A high degree of purification of L-amino acids is required for food products and academic use and the purity needs to be confirmed in the production lines. Therefore, it is important

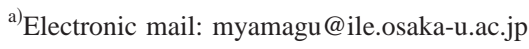

to develop a method to determine the amounts of the enantiomers and the racemic compound of amino acids. However, the currently used methods for measuring enantiometric purity are time consuming and lack in sufficient precision. ${ }^{12}$ Methods based on absorption spectra in the $\mathrm{THz}$ range are possible ways to distinguish between the enantiomers and the racemic compound because the absorption bands in the $\mathrm{THz}$ range are sensitive to the difference in the crystal structures. ${ }^{13}$ The $\mathrm{THz}$ time domain spectroscopy $(\mathrm{THz}-\mathrm{TDS})^{14}$ using the THz pulses generated by femtosecond laser pulses is a powerful tool for measuring the optical properties in the frequency range below $100 \mathrm{~cm}^{-1}(3 \mathrm{THz})$. Absorption spectra with a better signal-to-noise ratio can be obtained by the THz-TDS compared to those measured by the far-infrared Fourier transform (FIR FT-IR) spectroscopy in this range.

In this letter, we measured the absorption spectra of polycrystalline $\quad \mathrm{L}-, \quad \mathrm{D}-, \quad$ and $\quad$ DL-alanine $\left[\mathrm{H}_{3} \mathrm{~N}^{+}-\mathrm{CH}\left(\mathrm{CH}_{3}\right)-\mathrm{COO}^{-}\right]$by the THz-TDS. DL-Alanine forms the racemic compound and not the racemic mixture when crystallized from water solution. ${ }^{15}$ The measurement of the $\mathrm{THz}$ spectrum has not been reported about DL-alanine although L-alanine has been studied in detail by the FIR FT-IR spectroscopy. ${ }^{16}$ It was found that the THz absorption spectra show a large difference between the enantiomers and racemic compound of alanine. Based on this result, we propose a method to determine the enantiometric composition with the $\mathrm{THz}$ absorption spectra.

L-, D-, and DL-Alanine powders were purchased from Sigma-Aldrich and they were used without any further purification. We mixed alanine powder with $\mathrm{MgO}$ powder, the absorption coefficient of which is smaller than $20 \mathrm{~cm}^{-1}$ in the measured frequency range. The intensity of the continuous absorption due to the $\mathrm{MgO}$ additive was weaker than one fifth of that of alanine measured. We mixed $100 \mathrm{mg}$ alanine with 100 and $70 \mathrm{mg} \mathrm{MgO}$ to obtain the $\mathrm{THz}$ spectra in Figs. 1 and 2, respectively. These mixtures were pressed under a pressure of $14 \mathrm{MPa}$ to form pellets with a diameter of $13 \mathrm{~mm}$ and a thickness of $1 \mathrm{~mm}$. We measured the absorption spectra of the sample pellets in the frequency range from 10 to $90 \mathrm{~cm}^{-1}$ using a standard THz-TDS setup. ${ }^{17}$ A modelocked Ti:sapphire laser was used as the pump source, a 


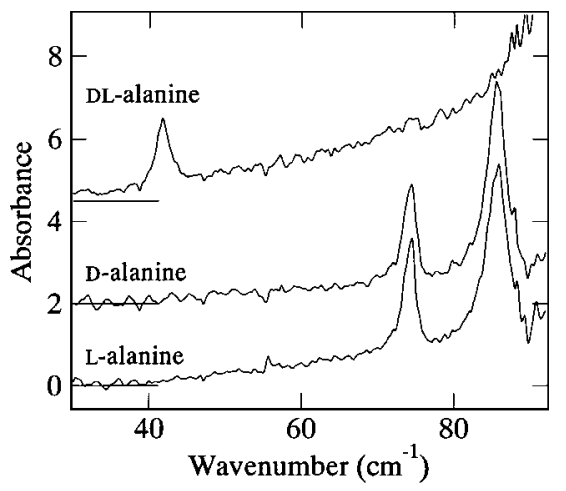

FIG. 1. THz absorption spectra of L-, D-, and DL-alanine. The absorption spectra of D- and DL-alanine are offset along the vertical axis.

pulse width and a repetition rate of which were $50 \mathrm{fs}$ and $82 \mathrm{MHz}$, respectively. The emitter was a 1-mm thick (110)cut $\mathrm{ZnTe}$ crystal excited by laser pulses with the average power of $160 \mathrm{~mW}$. The $\mathrm{THz}$ radiation from the emitter was collected and focused on the sample by a parabolic mirror. The $\mathrm{THz}$ radiation transmitted through the sample was collected and focused by another parabolic mirror on to the detector photoconductive antenna, which was a lowtemperature-grown GaAs-based antenna ${ }^{18}$ triggered by probe pulses with the average power of $15 \mathrm{~mW}$. The photoconductive current signal, which is proportional to the sampled $\mathrm{THz}$ field amplitude, was detected with a lock-in amplifier and its time-domain signal was obtained by scanning the optical delay of the probe pulse with respect to the pump pulse. By Fourier transforming the time-domain signal, we obtained the amplitude spectra, $E_{s}(\nu)$ and $E_{r}(\nu)$, for the sample and the reference pellets, respectively. The reference pellet contained only $\mathrm{MgO}$ with the same amount of the measured sample. The absorption spectra of a sample, $\alpha(\nu)$, were calculated using,

$$
\alpha(\nu)=-\ln \left|\frac{E_{s}(\nu)}{E_{r}(\nu)}\right|^{2}
$$

The spectral resolution of Figs. 1 and 2 is $0.13 \mathrm{~cm}^{-1}$.

Figure 1 shows the absorption spectra of L-, D-, and DL-alanine. All spectra show several absorption peaks and

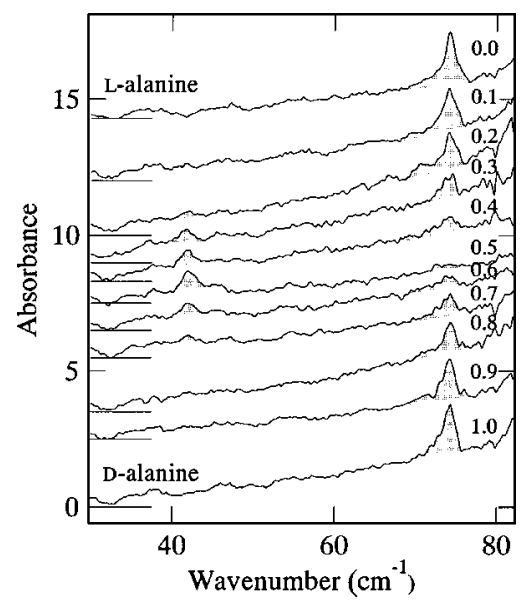

FIG. 2. Absorption spectra of the mixtures of the enantiomers (L- or $\mathrm{D}$-alanine) and the racemic compound (DL-alanine). The values represent the composition ratio $\rho$ of D-alanine in the sample before recrystallization. Each spectrum is shifted vertically for clarity.

Downloaded 17 Feb 2005 to 133.1.82.236. Redistribution subject continuous absorptions which increase with frequency. Two absorption bands are observed at 74.4 and $85.7 \mathrm{~cm}^{-1}$ for Land D-alanine. The two bands correspond to those appearing at 73 and $85 \mathrm{~cm}^{-1}$ in FIR spectra reported by Bandeker et $a l^{16}$ For DL-alanine, no absorption bands at 74.4 or $85.7 \mathrm{~cm}^{-1}$ but one absorption band at $41.8 \mathrm{~cm}^{-1}$ is observed. The band frequencies for L-(D-) alanine and that for DLalanine are quite different from each other. The difference of these peak frequencies suggests that these bands originate from the intermolecular vibrational modes. To confirm this, we measured the absorption spectra of the deuterated L-alanine, L-alanine- $d_{7}\left[\mathrm{D}_{3} \mathrm{~N}^{+}-\mathrm{CD}\left(\mathrm{CD}_{3}\right)-\mathrm{COO}^{-}\right]$. By deuteration, the absorption band observed at $74.4 \mathrm{~cm}^{-1}$ for L-alanine shifts to $72.7 \mathrm{~cm}^{-1}$ for L-alanine- $d_{7}$. The frequency of a vibrational mode is inversely proportional to the square root of the reduced mass. The frequency shift of the $74.4 \mathrm{~cm}^{-1}$ band can be explained by estimating the reduced mass associated with the vibrational mode. The observed shift of the peak frequency from $74.4 \mathrm{~cm}^{-1}$ for L-alanine agrees well with the calculated shift based on the assumption that the reduced mass of this vibrational mode is equal to the molecular weight of alanine ( 89 for L-alanine and 96 for L-alanine- $d_{7}$ ). Therefore, the agreement of the band shift indicates that the absorption bands observed in the measurement frequency range definitely originate from the intermolecular vibrational mode. The crystal structures of L- and D-alanine (enantiomers) are orthorhombic (the space group $D_{2}^{4}$ ) with four monomers in the unit cell, ${ }^{19}$ and that of DLalanine is orthorhombic (the space group $C_{2 v}^{9}$ ) with four monomers in the unit cell. ${ }^{15}$ Both structures are similar to each other in cell dimensions and projected structures. ${ }^{19}$ Nevertheless, the $\mathrm{THz}$ spectra are quite different from each other, suggesting their sensitiveness to a small change of the crystal structure.

The continuous absorption observed in all absorption spectra in Fig. 1 decreases with decreasing temperature, but does not disappear completely even at $10 \mathrm{~K}$ (not shown). The continuous absorption at $10 \mathrm{~K}$ is considered to be due to the scattering loss by the grains in the sample pellets since this background is sensitive to the grain size and the uniformity of the mixture of alanine and $\mathrm{MgO}$ powder. The temperature-dependent components of the continuous absorption are considered to have at least two contributions. One is the two-phonon processes, in which absorption due to sum and difference combinations of fundamental phonon modes occurs. ${ }^{20}$ Another contribution to the continuum is the tail of the absorption bands above $100 \mathrm{~cm}^{-1}$. There is the strong absorption band peaked at about $110 \mathrm{~cm}^{-1}$ for L-alanine at $10 \mathrm{~K}^{16}$ This band broadens and shifts to lower frequency with increasing temperature. The tail of this band is observed in the measured frequency range as the broad background absorption.

The large spectral difference between the enantiomers and the racemic compound in Fig. 1 makes it possible to determine the ratios of the racemic compound to the enantiomers in mixed samples. The quantitative determination of the composition ratio of alanine racemic compound to its enantiomers in a mixture is demonstrated by measuring the $\mathrm{THz}$ spectra of the mixtures with different composition of Land D-alanine. The samples, which were prepared by mixing L- and D-alanine in arbitrary ratios, were dissolved into pure water. After the recrystallization, the samples were pressed into pellets with $\mathrm{MgO}$ powder. The equal amounts of $\mathrm{L}-$ and o AlP license or copyright, see http://apl.aip.org/apl/copyright.jsp 


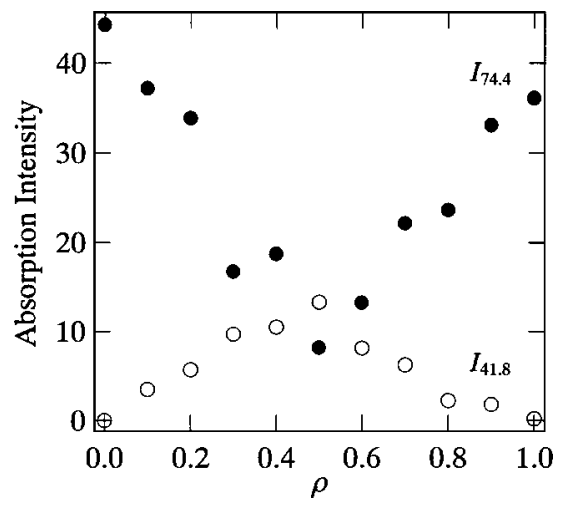

FIG. 3. Dependence of the absorption intensity of the bands at $41.8 \mathrm{~cm}^{-1}$ (open circles) and $74.4 \mathrm{~cm}^{-1}$ (closed circles) on the composition ratio of D-alanine.

D-alanine form DL-alanine in racemic compound and the excess of one of the enantiomers remains in the recrystallized samples. Figure 2 shows the composition dependence of the THz spectra. The values $\rho$ in Fig. 2 indicate the composition ratios of $\mathrm{D}$-alanine in the sample before mixing. The shaded area of each spectrum represents the absorption band of DLalanine at $41.8 \mathrm{~cm}^{-1}\left(I_{41.8}\right)$ and the enantiomers at $74.4 \mathrm{~cm}^{-1}$ $\left(I_{74.4}\right)$. The intensity $I_{41.8}$ becomes strongest when $\rho$ is 0.5 and vanishes when $\rho$ is 0.0 or 1.0 . On the contrary, $I_{74.4}$ becomes strongest when $\rho$ is 0.0 or 1.0 and almost disappears when $\rho$ is 0.5 .

Figure 3 shows the composition dependence of $I_{41.8}$ and $I_{74.4}$. The significant feature in Fig. 3 is the linear dependences of $I_{41.8}$ and $I_{74.4}$ on $\rho$. This linearity enables us to determine the composition ratio of the racemic compound to the enantiomers quantitatively from the ratio of $I_{41.8}$ to $I_{74.4}$. This result also suggests that $\rho$ in the sample can be determined when we know which of D- and L-alanine is the major content. The determination of $\rho$ based on the THz absorption can be applied to dating in the archaeology. Isomerization from L- to D-amino acids in living organisms starts just after the death and proceeds with long half-lives. ${ }^{21}$ We can estimate the date when a fossil animal died from $\rho$ in the following procedures. (1) Isolation and purification of alanine in fossils of animals. (2) Recrystallization of the mixture (Land D-alanine). (3) Measurements of the absorption bands at 41.8 and $74.4 \mathrm{~cm}^{-1}$ (or $85.7 \mathrm{~cm}^{-1}$ ) by the THz-TDS. (4) Determination of $\rho$ from the relative intensity of the absorption bands at 41.8 and $74.4 \mathrm{~cm}^{-1}$ ( or $85.7 \mathrm{~cm}^{-1}$ ).

The chemical analysis of polymorphs ${ }^{6}$ and racemic compounds using the THz-TDS is very effective for relatively pure materials. However, we should be aware of several fundamental problems, which limit the usefulness of the THzTDS for a wide range of applications. Absorption intensities of the intermolecular vibration are relatively weak compared to those of the intramolecular vibration and electronic transition in the mid-infrared and visible regions. The chemical analysis by the THz-TDS cannot be made for small amounts of samples though we can measure the THz spectra of such thick samples as do not transmit the mid-infrared and visible light inversely. Further, the strong absorption of water in the $\mathrm{THz}$ range makes it difficult to obtain the intrinsic spectra of molecules in water solution and mixed materials containing water. The THz-TDS would expand in real-world applications by overcoming these problems.

In summary, we have measured the absorption spectra of polycrystalline L-, D-, and DL-alanine by the THz-TDS. We found that the absorption peak frequency of DL-alanine is significantly different from those of L- and D-alanine. The similar THz spectral differences in peak frequencies between pure enantiomers and the racemic compounds are observed for the following amino acids: valine, leucine, isoleucine, aspartic acid, methionine, phenylalanine, tyrosine, proline, and tryptophan. These results indicate that the absorption bands in the THz range are sensitive to the crystal structures of amino acids. We proposed and demonstrated a method to determine the enantiometric composition in amino acids which form the racemic compounds by the THz spectroscopy. This method offers the possibility to provide a practical method for distinguishing between the enantiomers and racemic compounds in the pharmaceutical industry and in archaeological applications.

Part of this work has been supported by the Grant-in-Aid for Scientific Research from the Ministry of Education, Culture, Sports, Science, and Technology, Japan. This work has also been supported partly by Terahertz Optics Project for Medical Application led by J. Nishizawa, organized by Ministry of Education, Culture, Sports, Science, and Technology, Japan.

${ }^{1}$ M. Nagel, F. Richter, P. Haring-Bolivar, and H. Kurtz, Phys. Med. Biol. 48, 3625 (2003).

${ }^{2}$ R. M. Woodward, V. P. Wallace, R. J. Pye, B. E. Cole, D. D. Arnone, E. H. Linfield, and M. Pepper, J. Invest. Dermatol. 120, 72 (2003).

${ }^{3}$ K. Yamamoto, M. Yamaguchi, F. Miyamaru, M. Tani, M. Hangyo, T. Ikeda, A. Matsushita, K. Koide, M. Tatsuno, and Y. Minami, Jpn. J. Appl. Phys., Part 2 43, L414 (2004).

${ }^{4}$ K. Kawase, Y. Ogawa, and Y. Watanabe, Opt. Express 11, 2549 (2003). ${ }^{5}$ K. Yamamoto, M. Yamaguchi, M. Tani, M. Hangyo, S. Teramura, T. Isu, and N. Tomira, Appl. Phys. Lett. 85, 5194 (2004).

${ }^{6}$ P. F. Taday, I. V. Bradley, D. D. Arnone, and M. Pepper, J. Pharm. Sci. 92, 831 (2003).

${ }^{7}$ M. Hineno and H. Yoshinaga, Spectrochim. Acta, Part A 30, 411 (1974).

${ }^{8}$ M. Walther, P. Plochocka, B. Fischer, H. Helm, and P. Uhd Jepsen, Biopolymers 67, 310 (2002).

${ }^{9}$ Y. Fukami and K. Awazu, Jpn. J. Appl. Phys., Part 1 42, 3716 (2003).

${ }^{10}$ J. Nishizawa, Nihon Onkyou Gakkaishi 57, 163 (2001) [in Japanese].

${ }^{11}$ P. F. Taday, I. V. Bradley, and D. D. Arnone, J. Biol. Phys. 29, 109 (2003).

${ }^{12}$ C. D. Tran, V. I. Grishko, and D. Oliveira, Anal. Chem. 75, 6455 (2003), and references therein.

${ }^{13}$ M. Walther, P. Plochocka, B. Fischer, H. Helm, and P. Und Jepsen, Chem. Phys. Lett. 332, 389 (2000).

${ }^{14}$ D. Grischkowsky, S. Keiding, M. Exter and Ch. Fattinger, J. Opt. Soc. Am. B 7, 2006 (1990).

${ }^{15}$ M. S. Nandhini, R. V. Krishnakumar, and S. Natarajan, Acta Crystallogr., Sect. C: Cryst. Struct. Commun. 57, 614 (2001).

${ }^{16}$ J. Bandekar, L. Genzel, F. Kremer, and L. Santo, Spectrochim. Acta, Part A 39, 357 (1983).

${ }^{17}$ M. Hangyo, T. Nagashima, and S. Nashima, Meas. Sci. Technol. 13, 1727 (2002).

${ }^{18}$ M. Tani, S. Matsuura, and K. Sakai, Appl. Opt. 36, 7853 (1997).

${ }^{19}$ H. J. Simpson, Jr. and R. E. Marsh, Acta Crystallogr. 20, 550 (1966).

${ }^{20}$ M. Schall, M. Walther, and P. Uhd Jepsen, Phys. Rev. B 64, 094301 (2001).

${ }^{21}$ T. Lindahl, Nature (London) 365, 700 (1993). 